\title{
Innovación en la biblioteca pública española: dónde estamos y hacia dónde vamos
}

\author{
F. Javier GARCÍA GÓMEZ \\ Biblioteca Pública Municipal. Ayuntamiento de San Javier (Murcia) \\ Grupo de Investigación "Bibliotecas, Archivos y Cultura de la Información". Departamento de \\ Información y Documentación. Universidad de Murcia \\ fjgg3@um.es
}

Recibido: Septiembre 2012

Aceptado: Enero 2013

Resumen: La innovación, elemento fundamental en el momento histórico actual, resulta primoridal para que instituciones de todo tipo afronten con garantías los retos que se les plantean. Generalmente, estos procesos de innovación se basan fundamentalmente en el componente tecnológico, aunque también es posible llegar a innovar con otros recursos, planteamientos, y enfoques de servicio. La innovación también se está convirtiendo en una de las señas de identidad de las bibliotecas públicas españolas. Estas instituciones innovan principalmente a partir de la tecnología disponible ofreciendo servicios personalizados y adaptados en función de las necesidades e intereses de su comunidad de usuarios. Se analizan algunos casos paradigmáticos de servicios innovadores en bibliotecas públicas españolas.

Palabras clave: Bibliotecas Públicas, Innovación, Innovación Tecnológica, Creatividad, España, Servicios a la Comunidad, Servicios basados en Internet

\section{Innovation in the Public Library: where we are and where we go}

\begin{abstract}
Innovation, a key element in the current historical moment, it primoridal for all institutions face up to the challenges guarantees they face. Generally, these innovation processes are based primarily on the technological component, although it is possible to innovate with other resources, approaches, and service approaches. Innovation also is becoming one of the hallmarks of Spanish public libraries. These institutions mainly innovate from available technology offering personalized and adapted according to the needs and interests of its user community. It analyzes some paradigmatic cases of innovative services in Spanish public libraries
\end{abstract}

Keywords: Public Libraries / Innovation / Technological Innovation / Creativity / Spain / Community Services / Internet Based Services

"Las especies que sobreviven no son las más fuertes ni las más inteligentes, sino aquellas capaces de responder mejor a los cambios del medio" ${ }^{1}$

\footnotetext{
${ }^{1}$ Charles Darwin (1859)
} 


\section{INTRODUCCIÓN}

¿Pueden considerarse a las bibliotecas públicas españolas como innovadoras?, ¿Qué significa que una biblioteca sea innovadora? ${ }^{2}$ Preguntas de este tipo nos plantearemos en este texto e intentaremos ofrecerles la mejor respuesta posible. La innovación, elemento fundamental en la época actual, un entorno tan cambiante como en el que vivimos hoy en día, es un concepto sencillo y complejo a la vez ${ }^{3}$. Tradicionalmente, la innovación se la ha asociado de manera directa con la tecnología presentándose como un componente importante de cualquier proceso de innovación, aunque no sea considerada totalmente imprescindible (Echeverría, 2008). En este sentido, la innovación también se consigue a partir de la filosofía de trabajo que impregna los cambios en los modelos de gestión y evaluación de servicios (Lozano, 2008), en proyectos de colaboración interdisciplinar (Knecht, 2008), o en los sistemas de trabajo que buscan la orientación y satisfacción del ciudadano (Romera, 2008), entre otros.

La innovación aporta un valor añadido y ofrece nuevas soluciones a problemas o necesidades concretas. En este contexto, la innovación se concibe como una apuesta por el cambio. La innovación se alimenta de la búsqueda de nuevos puntos de vista en la resolución de un problema o una necesidad, haciendo uso de la imaginación, la inventiva, la creatividad y el flujo de ideas apoyándose en la experiencia previa existente así como en las habilidades personales y sociales de las personas o instituciones implicadas en el proceso.

No obstante lo dicho, aunque el desarrollo de las habilidades mencionadas permiten afrontar con garantías situaciones que necesitan de una resolución creativa y original, la realidad y la experiencia nos indica que la gestión de la tecnología ocupa un lugar primordial para obtener resultados exitosos, marcando la diferencia y refrescando la modalidad de servicios ofertados (Jantz, 2002). Así sucede también en la labor que desarrollan las bibliotecas públicas, entidades que cumplen un importante rol social en su comunidad, con capacidad para conectar con su territorio, utilizando recursos tecnológicos y no tecnológicos como fuente de innovación en los servicios.

2 González, Nieves: "¿Consideras que tu bilioteca es innovadora? Compruébalo" [En línea]. Bibliotecarios 2.0 [Fecha de publicación: 21-07-2011] http://www.nievesglez.com/ 2011/07/consideras-que-tu-biblioteca-es.html

3 El concepto de innovación ha sido definido de múltiples formas y con enfoques diferentes sin existir un consenso en este sentido. Teniendo en cuenta que la innovación es multifacética y que la misma va adaptándose a la sociedad, al tiempo que abarca diversas dimensiones y varía con el paso del tiempo, es necesario revisar periódicamente su significado e interpretación. Una relación de definiciones sobre innovación puede consultarse en http://www.funandi.edu.co/funandi/Seminario/memorias/S1DefinicionesdeInnovacion.pdf 
Como cambio e innovación van íntimamente relacionadas, la gestión del cambio aplicada al trabajo bibliotecario ya supone de por sí innovación (Lozano, 2009), ya que la predisposición a cambiar es necesaria para innovar, y al contrario: uno de los soportes de la gestión del cambio en las bibliotecas se encuentra en la innovación, pero al mismo tiempo, la innovación es el resultado de todo proceso de cambio bibliotecario (Gallo, 2008). En este sentido, somos de la opinión de que la innovación en bibliotecas, ya se encuentre basada o no en la tecnología, ayuda a superar estereotipos que la sociedad asocia a las mismas ${ }^{4}$.

En este artículo dejaremos constancia de que la innovación es una de las señas de identidad de las bibliotecas públicas españolas. Podremos comprobar que estas instituciones innovan principalmente haciendo uso de la tecnología como un medio -no como un fin en sí mismo-, llegando a ofrecer servicios personalizados y adaptados en función de las necesidades e intereses de su comunidad de usuarios. También ofreceremos algunos ejemplos de innovación bibliotecaria basada en la gestión de servicios, en los enfoques de trabajo, y en la orientación al usuario.

\section{INNOVACIÓN Y BIBLIOTECAS PÚBLICAS: ¿QUÉ CAMINO TOMAR?}

"(...) los escollos materiales con los que a menudo nos
encontramos al intentar conseguir aumentos en las
dotaciones presupuestarias y de personal son -a pesar de
todo- más fáciles de superar que las barreras invisibles
de nuestras conciencias, que nos mantienen aferrados a
modelos tradicionales de biblioteca"

Debemos comprender que la razón de ser de las bibliotecas son fundamentalmente los usuarios, más que la información en sí. Partiendo de este requisito ineludible, el trabajo bibliotecario de carácter innovador, creativo y competitivo debe guiarse bajo este prisma. Lo importante es lo que quiere el usuario, no lo que nosotros pensamos que quiere. A partir de aquí, si queremos innovar y sorprender, es necesario adaptarnos a nuestro entorno, a las necesidades e intereses de los usuarios, para ofrecerles lo que

4 Un ejemplo es el ruido. Las bibliotecas silenciosas, las bibliotecas como templos del silencio, han pasado a mejor vida; ahora el ruido es (debe ser) parte de la dinámica diaria de las bibliotecas, siempre moderadamente. El ruido implica que las bibliotecas han cambiado, son lugares llenos de vida, donde las gentes se encuentran, intercambian opiniones, se relacionan y se integran. Son lugares que invitan a los ciudadanos a utilizarlas, a participar y formar parte de ellas. Esa es otra gran innovación (cambio) que experimentan actualmente cada vez más bibliotecas, a pesar de que a determinados profesionales bibliotecarios les cuesta "digerir" este nuevo devenir de las cosas

5 Domínguez Sanjurjo, Ramona: "La biblioteca pública y los servicios de información a la comunidad" [En línea]. Educación y Biblioteca, $\mathrm{n}^{\circ} 135$ (2003), página 51 http://gredos.usal.es/jspui/bitstream/10366/108874/1/EB15_N135.pdf 
realmente quieren y cómo lo quieren. El resultado será, de cara a nuestra comunidad, unas bibliotecas prácticas, útiles y atractivas.

En el Documento Final del V Congreso Nacional de Bibliotecas Públicas (Gijón, $2010)^{6}$ se abogaba por "evitar el exceso de reglamentaciones de uso y normativas que anquilosan e inciden en los tópicos más recurrentes sobre la idea de la biblioteca pública". En este mismo documento se destacaba "como valor básico en la biblioteca pública actual la diversión y la creatividad, ahuyentar el aburrimiento". Y se incidía en la idea de "dar protagonismo a la intuición y cuestionar ideas preestablecidas, huir del conformismo, combatir la imagen tipificada de la biblioteca y de la profesión para transformarla en el imaginario popular".

Llegados a este punto cabe hacerse la pregunta clave: ¿Son las bibliotecas públicas españolas innovadoras?, ¿Es posible encontrar propuestas innovadoras en su gestión, en sus enfoques de trabajo, en los servicios que ofrecen a los usuarios, en definitiva en su modus operandis? Nosotros somos de la opinión de que sí, muchas bibliotecas públicas del Estado español están adoptando nuevos métodos y estrategias de trabajo que pueden ser consideradas innovadoras y es objeto de este trabajo dar muestras de ello.

\section{LAS BIBLIOTECAS PÚBLICAS ESPAÑOLAS TAMBIÉN INNOVAN}

“(...) la profesión de bibliotecario se ha distinguido siempre por su capacidad y disposición para estar alerta ante las innovaciones de todo tipo que puedan suponer un mejor servicio a su comunidad",

Es posible identificar actividades, recursos y servicios innovadores en las bibliotecas públicas españolas, ya que generan valor mejorando por ejemplo la alfabetización informacional de su comunidad, el nivel educativo de los miembros de su localidad o bien ofreciendo información de calidad y actualidad sobre temas de interés para los ciudadanos.

Las bibliotecas públicas españolas han innovado en practicamente todos los terrenos del trabajo bibliotecario: organizando servicios, planificando y organizando sus colecciones de recursos de información, aplicando técnicas de marketing, poniendo en marcha programas de actividades que persiguen facilitar la integración social y laboral de sus destinatarios, prestando materiales a domicilio con nuevas aplicaciones o sistemas, haciendo uso de las tecnologías de la información para llegar mejor a los usuarios tanto reales como potenciales, etc. Y en el trasfondo de estas

6 Disponible en http://www.mcu.es/bibliotecas/docs/MC/2010/CongresoBP/Documento finalVCNP.pdf

7 Pasadas Ureña, Cristóbal: “Competencias básicas, aprendizaje permanente y bibliotecas públicas. Experiencias de ámbito nacional e internacional”. En: La acción social y educativa de la biblioteca pública en tiempos de crisis. Murcia: Tres Cultura, 2010; 65-91 
iniciativas de trabajo aparece siempre de manera destacada la figura del profesional bibliotecario como impulsor y dinamizador de las mismas.

Estas experiencias innovadoras tienen aún mucho más mérito en el actual contexto de restricciones económicas en el que nos encontramos. Significa que muchos bibliotecarios han decidido ver la crisis económica más como una oportunidad que como una amenaza (que también lo es), un medio para justificarse ante la sociedad, ante sus usuarios y ante los responsables políticos para que sigan apoyando y apostando por la biblioteca pública. Porque sí, se pueden ofrecer buenos y renovados servicios aún en un contexto de recesión económica (García y Díaz, 2011). La tarea es más difícil, pero los resultados son más satisfactorios y reconfortantes a nivel personal y profesional. La crisis puede verse entonces como un reto, un desafío profesional, un elemento de superación.

En este texto destacaremos el trabajo de determinadas bibliotecas que son innovadoras porque reinventan servicios y funciones, prueban nuevas actividades, experimentan, se equivocan, y corrigen errores. En definitiva, son bibliotecas que innovan, que crean, que inventan, que marcan pautas, que marcan la diferencia. Son bibliotecas en las que a falta de grandes recursos económicos, materiales y humanos, van sobradas de ideas, de capital intelectual, de ganas de sacar adelante esto como sea, son bibliotecas que buscan y consiguen la sorpresa entre sus usuarios, son bibliotecas -una vez más- innovadoras. Y si nos fijamos bien, junto a la tecnología como elemento destacable en muchas de ellas, se une el trabajo de planificación realizado, la gestión eficaz en su desarrollo y el éxito obtenido.

Las bibliotecas publicas españolas son innovadoras y muestra de ello son las experiencias de trabajo que aquí presentaremos. Estos procesos de innovacion pueden encuadrarse, bajo nuestro punto de vista, en tres grandes categorias: innovacion basada en tecnologias de la informacion y de la participacion, innovacion como soporte a su funcion sociocultural, e innovacion en cuanto a su implicacion con la comunidad.

\section{Innovación basada en tecnologías de la información y de la participación}

Las bibliotecas ofrecen servicios innovadores basándose en las tecnologías de la información y comunicación (TIC) y en las denominadas tecnologías de la participación (TP $)^{8}$. Son servicios que se prestan presencial y remotamente y en los que los recursos tecnológicos son fundamentales para el correcto desarrollo del servicio. Son servicios basados pues en herramientas tecnológicas que ofrecen un valor añadido a la labor bibliotecaria.

Servicios de autopréstamo y circulación documental. La irrupción de las TIC en el entorno bibliotecario tuvo como una de las primeras aplicaciones la gestión de

\footnotetext{
${ }^{8}$ Merlo Vega, José A.: "Las tecnologías de la participación en la biblioteca" [En línea]. Educación y Biblioteca, No161 (2007), 61-68 http://eprints.rclis.org/handle/10760/10558
} 
la circulación documental. Se introdujeron sistemas automatizados de control documental que gestionaban la circulación de documentos en una biblioteca: el alta en la base de datos y su incorporación al catálogo automatizado, la entrada y salida de documentos en la biblioteca, el préstamo y su devolución, y últimamente las reservas y renovaciones de préstamos documentales. Cuando la gestión de la circulación documental se realizaba in situ, es decir, en las instalaciones de la biblioteca siempre era necesaria la presencia humana (tanto del profesional bibliotecario como del usuario) y siempre dentro de su horario de servicio. Hoy en día algunas bibliotecas ofrecen al usuario la posibilidad de gestionar sus trámites de circulación documental (préstamos, devoluciones, reservas, renovaciones,....) sin la presencia física del bibliotecario. La implantación de sistemas autopréstamo y devolución documental, de renovaciones y reservas de documentos prestados comienzan a introducirse en el ámbito de las bibliotecas públicas. Queremos destacar aquí el caso de la biblioteca pública "Luis Martín Santos" de Vallecas (Madrid), pionera en este sentido. En 2008, la biblioteca se convierte en un centro de última generación al contar con un sistema de Identificación por Radiofrecuencia (RFID) ${ }^{9}$ que le permite la identificación, localización automática y autopréstamo a través de una etiqueta adherida al material de la biblioteca, que transmite ondas de radio con la identidad de cada documento ${ }^{10}$. Otras bibliotecas públicas españolas con sistemas de control documental por radiofrecuencia son la Biblioteca Pública del Estado de Ciudad Real, la Biblioteca Pública del Estado de Santiago de Compostela, o la Biblioteca Municipal Torreste Ballester de Salamanca entre otras.

Servicios de información y referencia online. Seguramente el servicio bibliotecario más propicio a ofrecerse en modo telemático. De hecho, proliferan en todo tipo de bibliotecas versiones digitales del tradicional servicio de información bibliográfica y referencia bibliotecario. El servicio en su versión virtual ha evolucionado al ritmo que evolucionaban las tecnologías y hoy en día en posible encontrar diferentes modalidades de prestación del mismo: por email, por formulario electrónico, por mensajería instantánea, por chat, en tiempo real, etc. ${ }^{11}$ En el ámbito de las bibliotecas públicas españolas, el servicio de referencia online por antonomasia es el que se ofrece desde Pregunte, las bibliotecas responden ${ }^{12}$.

${ }^{9}$ Salvador Esteban, Ana Julia: "Experiencia de la Biblioteca Luis Martin Santos de Villa de Vallecas amb el sistema de Radiofrequencia (RFID)" [En línea]. ITEM, n52 (2010), 90-97 http://www.raco.cat/index.php/Item/article/view/195682/331085

${ }^{10}$ Vídeo informativo sobre el sistema de autopréstamo, devolución documental y control por radiofrecuencia disponible en https://www.youtube.com/watch? $\mathrm{v}=$ FLvJgxVw48

${ }^{11}$ Merlo Vega, J. A.: "Referencia digital: concepto, tecnologías e implementación en centros de información" [En línea]. El Profesional de la Información, vol.18, nº (2009), 589-599 http://gredos.usal.es/jspui/bitstream/10366/70659/3/DBD_Referencia_digital.pdf

$12 \mathrm{http}: / /$ www.pregunte.es/ 
Pregunte... es el servicio de referencia virtual que ofrecen de manera colaborativa varias bibliotecas públicas españolas. Inicialmente ofrecía al usuario la posibilidad de realizar sus consultas a través de un formulario electrónico que trasladaba la consulta a la/s biblioteca/s "de servicio" y en un plazo máximo de 3 días se compromete el servicio a dar respuesta. Actualmente, es posible realizar esta consulta en tiempo real (vía chat) en un horario determinado por el servicio. Otras de las novedades es la posibilidad de realizar la novedad en varios idiomas (castellano, catalán, gallego, euskera e inglés). El servicio utiliza como garantía de su eficacia el lema Si tienes una pregunta, ya tienes una respuesta ${ }^{13}$. Otra de las novedades del servicio son los recursos de información en línea que complementan y/o amplian información a la proporcionada por el servicio a la consulta planteada por el usuario. El sistema aporta información sobre los recursos más valorados por los usuarios, los recursos más vistos, los descriptores más utilizados, un índice de materias, etc. También se están desarrollando sistemas de información online sobre temas y cuestiones de ámbito territorial, un servicio de referencia online local o regional. Serían, por ejemplo, el caso del servicio $L a$ Biblioteca Responde ${ }^{14}$ que ofrece la Red de Bibliotecas Públicas de la Junta de Andalucía en donde se da respuesta a las cuestiones que llegan sobre temas andaluces. A nivel local,esto es, sistemas de información y referencia sobre cuestiones de la localidad, municipio o comarca, podemos destacar los servicios ofrecidos por las Bibliotecas Municipales de A Coruña y la Red de Bibliotecas Públicas de la Diputación de Barcelona. En el primer caso, el servicio se denomina Pregunte por A Coruña ${ }^{15}$ en donde se da respuesta a preguntas sobre historia, personajes, o hechos ocurridos en la ciudad coruñesa, así como información bibliográfica sobre temas locales. En el caso barcelonés, Pregunta a la biblioteca ${ }^{16}$ se trata de un servicio bien reciente de esta red bibliotecaria que aporta información y recursos de interés en línea que tengan que ver con Barcelona y su demarcación territorial.

Alertas informativas. Como complemento a los servicios de referencia virtual, algunas bibliotecas ofrecen también alertas informativas en línea sobre temas de interés de los usuarios. Para ello, el usuario previamente debe darse de alta en el sistema de información de la biblioteca indicándole sus temas o eventos de interés. La biblioteca, entonces, periódicamente mantendrá informado al ciudadano según su perfil de usuario y en base a sus intereses. Queremos destacar en este sentido el

${ }^{13}$ Cuadrado, M ${ }^{\text {a }}$ Isabel y Rivera Díaz, Eva: "Pregunte: las bibliotecas responden. Servicio de referencia virtual de las bibliotecas públicas españolas". El Profesional de la Información, vol.18, nº (2009), 642-648

${ }^{14}$ Disponible en http://www.juntadeandalucia.es/cultura/ba/responde/

${ }^{15}$ Disponible en http://www.coruna.es/bibliotecas

${ }^{16}$ Disponible en http://bibliotecavirtual.diba.cat/es/pregunta-a-la-biblioteca 
servicio de alertas informativas proporcionado por las Bibliotecas Municipales de A Coruña vía SMS, esto es, mensajes a teléfonos móviles ${ }^{17}$. Este servicio es totalmente gratuito para el usuario e informa al usuario de actividades, novedades, nuevas incorporaciones, talleres formativos, peticiones realizadas, etc. Este servicio de alerta SMS ahorra tiempo a la biblioteca a la hora de localizar al usuario y supone una intromisión "menor" porque no requiere de respuesta por parte del usuario. El sistema genera unos 20 mensajes diarios ${ }^{18}$

Servicios de información local. Una extensión o especialización de los servicios de información y referencia virtual lo constituyen los servicios bibliotecarios que proporcionan información de interés y alcance local. En cierto modo, pueden coincidir con los servicios de referencia local comentados, pero un servicio de información local debe abordar compromisos y un alcance más amplio, tanto en calidad como en cantidad de información. Lo ideal es la constitución de un portal web que aglutine todos los recursos de información de temática local. En nuestro país el servicio de información local online por excelencia es el que viene ofreciendo desde 2002 la Biblioteca Pública del Estado de Tarragona ${ }^{19}$. El sistema responde a preguntas sobre Tarragona, ofrece también respuesta a consultas bibliográficas y ofrece guías prácticas y orientativas para colectivos de Tarragona: mayores, inmigrantes, recién llegados, etc ${ }^{20}$. Las bibliotecas públicas han desarrollado y desarrollan también interesantísimas colecciones de estudios y temas locales que, con la ayuda de las TIC, las trasladan al universo web y universalizan el conocimiento, consulta y acceso a los materiales que las componen. Las colecciones y secciones de información local de las bibliotecas posiblemente sean uno de los servicios bibliotecarios con mayor proyección en la era digital ${ }^{21}$. Así queda de manifiesto en el sitio web que ha puesto en marcha la Biblioteca Municipal de Ermua (Vizcaya) ${ }^{22}$,seguramente uno de los mejores portales web dedicado a la sección local de la biblioteca. Esta innovadora sucursal web de biblioteca local ocupado un lugar destacado en el último Congreso Nacional de Bibliotecas Públicas

\footnotetext{
${ }^{17}$ Disponible en http://www.coruna.es/bibliotecas/

${ }^{18}$ Pesquero Murillo, Julio: "La biblioteca en el teléfono móvil" [En línea]. IV Congreso Nacional de Bibliotecas Públicas (A Coruña, 2008) http://travesia.mcu.es/portalnb/jspui/ handle/10421/555

${ }^{19}$ Disponible http://cultura.gencat.net/bpt/sil/index.htm

${ }^{20}$ Lozano, Roser: "Recursos virtuales y la comunidad local: el ejemplo de la Biblioteca Pública del Estado en Tarragona" [En línea]. IV Encuentro del Foro NAPLE (Madrid, 2007) http://www.mcu.es/bibliotecas/docs/MC/NAPLE/Recursos_Virtuales.pdf

${ }^{21}$ García Gómez, Francisco Javier: "Colecciones locales en bibliotecas públicas españolas: una mirada a sus espacios webs" [En línea]. V Congreso Nacional de Bibliotecas Públicas (Gijón, 2010) http://travesia.mcu.es/portalnb/jspui/bitstream/10421/4912/1/ FranciscoJavierGarcia.pdf

${ }^{22}$ Disponible en http://www.ermua.es/pagsbiblio/fondo_local/ca_index.asp
} 
celebrado en Burgos ${ }^{23}$. En esta misma línea innovadora se sitúan los sitios web dedicados a su sección local que ofrecen la Biblioteca Municipal de Vila-Seca (Tarragona) ${ }^{24}$ o la Biblioteca Municipal de Manlleu (Barcelona) $)^{25}$.

Servicios bibliotecarios basados en la Web 2.0. La Web 2.0 envuelven día a día cada vez más toda la esencia de los servicios bibliotecarios. Cada vez es más habitual encontrarnos a bibliotecas de todo tipo -y las públicas no son ninguna excepción- haciendo uso de las herramientas y aplicaciones que facilitan la web 2.0 o web social. El objetivo es poner al usuario en el centro de actuación de toda iniciativa bibliotecaria, solicitando y fomentando su participación, convirtiendo al mismo de mero consumidor a protagonista activo en este nuevo ámbito de acción bibliotecaria. Actualmente, el trabajo de bibliotecas públicas españolas en redes sociales, blogs, wikis, microblogs, y demás aplicaciones 2.0 es habitual consiguiendo ofrecer servicios en muchos casos realmente imaginativos y sugerentes. Si tuviéramos que destacar a alguna o algunas bibliotecas públicas de nuestro entorno, no podemos empezar sin olvidar a la Biblioteca Municipal de Muskiz (Vizcaya) pionera en el Estado español en el uso de la web 2.0 en la gestión bibliotecaria. Esta biblioteca ha servido de referente y guía para el resto de bibliotecas españolas que poco a poco iban subiéndose al carro de lo 2.0. Así, han surgido servicios bibliotecarios 2.0 ciertamente innovadores que sirven de reflejo de los beneficios de esta mezcla de tecnología, filosofía de trabajo y actitud en la gestión bibliotecaria y que aportan un matiz novedoso a los mismos. Algunos ejemplos de lo que comentamos son:

- El blog del club de lectura "El grito" de la Biblioteca Pública del Estado de Albacete $^{26}$. El blog -especializado en literatura de género (novela negra, aventuras, ciencia-ficción,...) comenzó su andadura por 2005 y actualmente se complementa con otros servicios 2.0 como son Facebook y Twitter $^{27}$. Las Bibliotecas Municipales de San Javier (Murcia) ${ }^{28}$ también utilizan diferentes blogs como medio de fomento de la lectura ${ }^{29}$. Destacamos el blog correspondiente al concurso de microrrelatos ${ }^{30}$ que desde 2.010, anualmente convocan y que cuentan con el patrocinio de un

${ }^{23}$ Información disponible en http://www.mcu.es/bibliotecas/MC/2012/CongresoBP/ index.html

${ }^{24}$ Disponible en http://www.bibliotecavila-seca.cat/

${ }^{25}$ Disponible en http://www.bibliotecamanlleu.cat/fedancius/

${ }^{26}$ Disponible en http://clubelgrito.blogspot.com.es/

${ }^{27}$ Prieto García, Juan A.: "Efectividad de las herramientas de la web social en un club de lectura. El caso del club de lectura El Grito" [En línea]. V Congreso Nacional de Bibliotecas Públicas (Gijón, 2010) http://travesia.mcu.es/portalnb/jspui/handle/10421/4943

${ }^{28} \mathrm{http}$ ///www.bibliotecaspublicas.es/sanjavier

${ }^{29}$ Disponibles en http://www.bibliotecaspublicas.es/sanjavier/agenda_lit.htm

${ }^{30}$ Disponible en http://microrrelatate.blogspot.com/ 
hotel de la zona. Actualmente, el blog se ha convertido en una inmensa base de datos de microrrelatos que permite el acceso a más de 1.000 pequeños relatos literarios

- Otra aplicación 2.0 realmente interesante son los wikis, espacios web producto del trabajo colaborativo llevado a cabo por diferentes personas. Estos espacios web suelen tratar temas concretos que van perfeccionándose e incrementándose con las portaciones de los colaboradores. Su máximo exponente han sido las enciclopedias, en concreto la Wikipedia, auténtico icono del movimiento web 2.0, ya que en este sitio web podemos ver representadas todas las carcaterísticas y funcionalidades que se reconocen a la web 2.0. En el ámbito de las bibliotecas públicas españolas aún no está muy extendido el uso de wikis para la creación y prestación de servicios. No obstante, sí es posible identificar ya algunas experiencias bibliotecarias exitosas que utilizan esta aplicación a modo de enciclopedia de alcance local, también conocidas con el nombre de localpedias o locapedias ${ }^{31}$. Así, podemos destacar de manera especial la wiki sobre temas locales puesta en marcha por las Bibliotecas Municipales de A Coruña ${ }^{32}$. En esta Web el usuario puede encontrar información sobre los personajes y entidades más destacados de la ciudad de A Coruña a lo largo de su historia. Asimismo, ofrece al usuario la oportunidad de colaborar y aportar contenidos, crear nuevos personajes y entidades que tengan una especial relación con la ciudad, etc. Otro servicio wiki destacable es el que ofrecen la red de bibliotecas públicas de Euskadi, utilizada en este caso como plataforma de formación para bibliotecarios municipales de la región ${ }^{33}$

- También los escritorios virtuales o páginas de inicio ${ }^{34}$ están aportando valor innovador al uso que de la web 2.0 hacen las bibliotecas públicas. Estos espacios web permiten a la biblioteca generar "universos temáticos" en los que se recopilan enlaces de interés a recursos de información sobre temas concretos, se ofrece información sobre las actividades de la biblioteca, se crean guías temáticas, se perfilan grupos de interés, se recopilan documentos de referencia, noticias de interés local, etc. Muy sugerentes son las propuestas que ofrecen, en esta sentido, la Biblioteca

31 Gómez Hernández, José A.: "Wikipedias y bibliotecas públicas. Participar en la información local digital a través de localpedias" [En línea]. Notas ThinkEpi 2011 (02 de Diciembre de 2.010) http://www.thinkepi.net/wikipedias-y-biblioteca-publica-participar-en-lainformacion-local-digital-a-traves-de-localpedias $\% \mathrm{E} 2 \% 80 \% 9 \mathrm{D}$

${ }^{32}$ Disponible en http://estudoslocais.wikispaces.com/

${ }^{33}$ Disponible en http://liburutegiak.wikispaces.com/

${ }^{34} \mathrm{http} / / /$ www.universoabierto.com/1323/la-biblioteca-20-netvibes-aplicacion-a-bibliotecas/ 
Municipal de Andorra (Teruel) ${ }^{35}$ o la Biblioteca Municipal de Burgos ${ }^{36}$, éste último ofertado como servicio de referencia en línea ${ }^{37}$

- Otras de las aplicaciones 2.0 que más enganchan en los últimos tiempos a las bibliotecas públicas son las denominadas redes sociales a través de las cuales, estas instituciones ofertan una gran variedad de servicios, aunque generalmente centran su acción por este medio como espacio publicitario, como tablón de anuncios, como plataforma de captación y fidelización de usuarios, y como no, como plaza pública en la que buscan la complicidad del usuario, fomentan su participación en los temas tratados, y generan flujos de conexión entre ellos y a partir de ellos. FaceBook ${ }^{38}$ es hoy en día la red social más utilizada y en donde es posible localizar más bibliotecas públicas $^{39}$. Podríamos dar muchos ejemplos de bibliotecas sociales, pero destacaremos aquí los casos de la Biblioteca Municipal de Piedras Blancas $\left(\right.$ Asturias) ${ }^{40}$ y las Bibliotecas Municipales de Barcelona, ${ }^{41}$ bibliotecas con dinamismo y muy activas en redes sociales

- Otro servicio 2.0 que últimamente está aflorando entre las bibliotecas públicas y que como en la mayoría de casos en nuestro país fue pionera la Biblioteca Municipal de Muskiz, es el de geolocalización, aplicación web que permite relacionar un recurso digital con una localización geográfica física, y utilizado principalmente como complemento de actividades de fomento de la lectura y de dinamización cultural:una de las más recurrentes son las denominadas rutas literarias 2.0. Destacamos por su interés y utilidad los paseos literarios de las Bibliotecas Municipales de Burgos $^{42}$ y el mapa literario de la Biblioteca Municipal de Muskiz ${ }^{43}$.

${ }^{35}$ Disponible en http://www.netvibes.com/biblioteca62

${ }^{36}$ Disponible en http://www.netvibes.com/bibmunburgos

${ }^{37}$ El servicio se autodefine como "un escritorio virtual en el que se ofrecen numerosas fuentes de información, seleccionadas por el personal bibliotecario, de interés para todos aquellos que sientan curiosidad por Burgos o precisen de sus servicios bibliotecarios desde cualquier lugar del mundo" http://www.aytoburgos.es/biblioteca/noticias-y-avisos/escritorio-virtual

${ }^{38}$ Disponible en http://www.facebook.es

${ }^{39}$ García Giménez, Daniel: "Redes sociales. Posibilidades de Facebook para las bibliotecas públicas" [En línea]. BiD, no24 (2010) http://www.ub.edu/bid/24/garcia2.htm

40 Disponible página de Facebook en https://www.facebook.com/pages/Bibliotecap\%C3\%BAblica-municipal-de-Piedras-Blancas/49786887135; Zarabozo, Ana: "Realidad 2.0. Estrategias de uso de los Social Media a pie de mostrador" [En línea]. V Congreso Nacional de Bibliotecas Públicas (Gijón, 2010) http://travesia.mcu.es/portalnb/jspui/handle/10421/4946

${ }^{41}$ Disponible página de Facebook en https://www.facebook.com/BibliotequesBCN?ref=ts; Cabre Serra, Anna: "Biblioteques de Barcelona en las redes sociales" [En línea]. V Congreso Nacional de Bibliotecas Públicas (Gijón, 2010) http://travesia.mcu.es/portalnb/jspui/handle/ $10421 / 4908$

${ }^{42}$ Disponible en http://paseosliterariosburgos.wikispaces.com/

${ }^{43}$ Disponible en http://www.muskiz-liburutegia.org/mapalit.html 
Sedes web de bibliotecas públicas. Las bibliotecas públicas españolas han visto en Internet, en su espacio Web, una oportunidad y una aliciente para seguir ofertando servicios novedosos, útiles, servicios acordes con los tiempos que corren, servicios para una nueva ciudadanía más habituada lo digital. Así, hoy en día, en un gran porcentaje, es posible encontrar sucursales virtuales bibliotecarias en las que se informa sobre la actividad de cada biblioteca, de sus recursos y servicios, de sus novedades, etc. No obstante, esto que en un principio se vio como todo un cambio de rumbo en el mundo bibliotecario, una forma de estar a la última, ha evolucionado con el paso de los años y ahora ya no es suficiente con estar, sino que además hay que ofrecer y ofrecerse como los usuarios requieren. Las bibliotecas en Internet, al igual que en el plano presencial, también deben estar enfocadas al usuario. De ahí, que poco a poco se vaya extendiendo entre estas instituciones la necesidad de ofrecer servicios web basados en criterios de accesibilidad, usabilidad y navegabilidad adaptados a las circunstancias, intereses y necesidades de los usuarios. En esta línea de trabajo caminan las sedes web de un buen puñado de bibliotecas públicas españolas entre las que destacamos en este trabajo las Bibliotecas Regionales de Murcia $^{44}$ Cantabria $^{45}$ y Castilla-La Mancha ${ }^{46}$, entre otras.

Puntos de acceso a Internet. Finalmente, las bibliotecas públicas también han innovado convertiéndose en puntos imprescindibles para acceder a Internet y a toda la gama de recursos de información disponibles en la Red ofreciendo servicios de acceso público a la Red y prestando incluso ordenadores portátiles para su uso, ofreciendo conexión wifi como complemento al servicio de acceso a Internet, orientando y aportando recomendaciones en un uso práctico, ético y seguro de la Red, ofreciendo acciones formativas gratuitas abiertas a todos los colectivos y edades en alfabetización digital, etc. Serían muchos los ejemplos que podríamos apuntar aquí, pero nos quedaremos con el trabajo que viene desarrollándose en la red de bibliotecas públicas de Andalucía ${ }^{47}$ que en esta área han resultado muchas veces pioneras y señaladas como modelo a seguir ${ }^{48}$.

\section{Innovación como proyección de su función social y cultural}

Las bibliotecas públicas españolas han utilizado la innovación como vehículo articulador de su rol sociocultural. Han apostado por ofrecer nuevos servicios o

\footnotetext{
${ }^{44}$ Disponible en http://www.bibliotecaregional.carm.es/Biblioteca/

${ }^{45}$ Disponible en http://bcc.cantabria.es/

${ }^{46}$ Disponible en http://pagina.jccm.es/biblioclm/

${ }^{47}$ Disponible en http://www.juntadeandalucia.es/cultura/ba/red-bibliotecas-publicasandalucia.php

48 Gómez Gómez, Antonio Agustín: "Proyectos y servicios innovadores en la Red de Bibliotecas Públicas de Andalucía” [En línea]. El profesional de la información, vol. 17, n 2 (2008), 175-182 http://www.elprofesionaldelainformacion.com/contenidos/2008/marzo/06.pdf
} 
servicios renovados que pueden considerarse como innovadores por lo sugerente de su propuesta. Son bibliotecas que destacan por su diferenciación ${ }^{49}$ y flexibilidad ${ }^{50}$, por su adaptabilidad y su entorno privilegiado. Son bibliotecas que sorprenden porque no son consideradas como la "típica biblioteca" ${ }^{51}$. En esta línea pueden resaltarse los casos de la Biblioteca Municipal Río Segura de Murcia ${ }^{52}$, la Biblioteca Can Torró de Alcudia ${ }^{53}$, la Biblioteca Municipal CUBIT para Jóvenes de Zaragoza ${ }^{54}$, o la Biblioteca Municipal de Peñaranda de Bracamonte (Salamanca) ${ }^{55}$.

- La biblioteca Río Segura de Murcia ofrece amplios espacios y mucha luz natural, la visita está amenizada con música ambiental, y tanto el equipamiento en su conjunto como el mobiliario disponible le aportan un aire de modernidad y contemporaneidad que la distinguen en su entorno inmediato. La biblioteca Cubit, inaugurada en julio de 2010, es una biblioteca pensada y centrada en un colectivo específico de usuarios: los jóvenes cuyas edades oscilen entre los 16 y 25 años. La biblioteca se constituye pues en un espacio integral para jóvenes, un lugar de inspiración y expresión juvenil, con un diseño y decorado atractivos, que presenta espacios para el encuentro y el esparcimiento ${ }^{56}$

- A las bibliotecas de Peñaranda y de Alcudia no las vamos a descubrir aquí ni a sus métodos de gestión que son y han sido sinónimos de innovación, creatividad y modelos a seguir. La biblioteca de Peñaranda, insertada en un área rural, ha conseguido ser puntera en muchos aspectos de la gestión bibliotecaria y se ha convertido en "el armazón básico de un centro integral de servicios informativos, formativos y culturales, el Centro de Desarrollo Sociocultural, cuya contribución al desarrollo local, bien de manera implícita o a través de la participación formal en diversos planes, ha sido una constante desde la última década" ${ }^{57}$. La biblioteca de Alcudia

49 Gambles, Brian: "Nuevos espacios para nuevos usos" [En línea]. BiD, nº25 (2010) http://www.ub.edu/bid/25/gambles3.htm

${ }^{50}$ Gómez Hernández, José A.: "La flexibilización del modelo de biblioteca pública en España" èn línea]. Notas ThinkEpi 2011 (15/12/2010) http://www.thinkepi.net/laflexibilizacion-del-modelo-de-biblioteca-publica-en-espana

${ }^{51}$ Bonet Peitx, Ignasi: "Biblioteca Enric Miralles de Palafolls: unos libros y un sueño" [En línea]. BiD, n²5 (2010) http://www.ub.edu/bid/25/bonet2.htm

${ }_{52} \mathrm{http}: / / \mathrm{www} . \mathrm{rmbm} .0 \mathrm{rg} /$ bibliotecas/riosegura

${ }^{53} \mathrm{http}: / /$ www.cantorro.es/principal/index.php?i=esp

${ }^{54} \mathrm{http}: / /$ www.zaragoza.es/ciudad/educacionybibliotecas/bibliotecasmunicipales/cubit.htm

${ }^{55} \mathrm{http} / / / \mathrm{cds}$.fundaciongsr.com/seccion_menu.php?a $=110$

${ }^{56}$ García Fernández, Inmaculada y Bordonaba del Río, Pilar: "Del cubo al cubit. Nace en Zaragoza la primera biblioteca de España para jóvenes". Mi Biblioteca, n²3 (2010), 41-45 http://www.cai.es/Portal2006Files/UserFiles/File/Binder1.pdf

${ }^{57}$ Hernández Sánchez, Hilario: "Consideraciones sobre desarrollo y bibliotecas rurales. Y un ejemplo" [En línea]. III Encuentro Biblioteca y Municipio (Madrid, 2009) 
centrada especialmente en los más jóvenes, se constituye en un espacio de encuentro y comunicación con vocación de servicio lúdico socialmente integrador.

En general, son bibliotecas que favorecen la experimentación y que pueden servir como referentes para extrapolar a otras bibliotecas. Un buen ejemplo lo supone la Biblioteca Municipal de Castropol (Asturias) que en 2008 recibió el Premio a la Calidad e Innovación que anualmente convoca la asociación profesional SEDIC, cuyo trabajo se desarrolla en el ámbito rural y a la que se reconoció su amplia gama de servicios, la calidad de su fondo documental o su integración en su comunidad ${ }^{58}$. Otra forma de reconocer el buen hacer de una biblioteca es mediante la concesión de los certificados de calidad AENOR, los cuales, reflejan un trabajo de planificación y gestión bien realizado, eficaz y de utilidad para sus destinatarios. Los casos de la Biblioteca Regional de Murcia ${ }^{59}$ o de la Biblioteca Municipal de Ermua (Guipuzcoa) ${ }^{60}$ son buenas muestras de ello. Otro buen ejemplo es la Biblioteca Pública del Estado de Salamanca ${ }^{61}$ que experimenta e innova a la hora de presentar y organizar sus colecciones de documentos poniendo en práctica sistemas de organización documental novedosos como los centros de interés que se ajustan mejor a las necesidades informativas de los usuarios y facilitan la búsqueda y localización de los recursos que son de su interés. En esta misma línea trabajan sus colecciones físicas otras bibliotecas como la Biblioteca Pública Provincial de Almería ${ }^{62}$, la Biblioteca Municipal de Ansoain (Navarra) ${ }^{63}$, o las Bibliotecas Municipales de Gijón (Asturias) ${ }^{64}$.

Son bibliotecas que abogan por la flexibilidad en los espacios y en su normativa, son bibliotecas que presentan un equipamiento de gran calidad, con horarios de uso muy amplios. Son bibliotecas que amplian su acción al terreno de lo cultural, del ocio, de la educación, de las demandas de los usuarios, que respiran tecnología, que transmiten sensaciones de modernidad y contemporaneidad. Aunque realmente, una biblioteca del siglo XXI debería ser así y no constituir una excepción

\footnotetext{
http://travesia.mcu.es/portalnb/jspui/handle/10421/2176

${ }_{58}^{58}$ Más información en http://www.sedic.es/premio-calidad08-nota-prensa.pdf

${ }^{59}$ Véase http://www.20minutos.es/noticia/921472/0/

${ }^{60}$ Véase http://www.elcorreo.com/vizcaya/v/20110215/guipuzcoa/biblioteca-ermua-obtienecertificado-20110215.html

${ }^{61}$ Véase http://www.bibliotecas.jcyl.es/web/jcyl/BibliotecaSalamanca/es/Plantilla100/ $1284227470282 /$ _ _ I

${ }^{62}$ Véase http://Www.juntadeandalucia.es/cultura/opencms/export/bibliotecas/bibalmeria/ servicios/centroInteres.html

${ }^{63}$ Véase http://www.bibliotecaspublicas.es/ansoain/seccont_27187.htm

${ }^{64}$ Véase http://bibliotecas.gijon.es/page/3210-centros-de-interes
} 


\section{Innovación en cuanto a su implicación con la comunidad}

La biblioteca busca marcos de colaboración con su comunidad, llega a ella de diferentes modos, busca a sus usuarios potenciales no limitándose a esperar que lleguen a sus instalaciones. La biblioteca sale de sus cuatro paredes ${ }^{65}$ y aparece en lugares insospechados ofreciendo lo inesperado. La biblioteca busca y logra sorprender al usuario al ofrecerle de manera creativa servicios enfocados a todo tipo de usuarios. Las biblioplayas y bibliopiscinas ${ }^{66}$, las biblioparques $^{67}$, las bibliomercados $^{68}$, las biblioplazas, las bibliometro ${ }^{69}$, las bibliotren ${ }^{70}$, bibliotecas en hospitales, farmacias ${ }^{71}$ y centros de salud, en prisiones ${ }^{72}$, las bebetecas ${ }^{73}$, los servicios a domicilio ${ }^{74}$, son claros ejemplos de cómo la biblioteca pública expande

65 "Proyectos de servicio bibliotecario más allá del equipamiento estable". Monográfico coordinado por Ignasi Bonet, Ester Omella y Enric Vilagrosa [En línea]. Educación y Biblioteca, nº149 (2005),55-125 http://gredos.usal.es/jspui/bitstream/10366/108920/1/EB17_149.pdf

${ }^{66}$ Blanco Faura, Irene: "Bibliopiscinas y biblioplayas: refrescantes servicios de extensión cultural" [En línea] Post publicado en Bibliogtecarios (08/07/2011) http://www.biblogtecarios.es/ ireneblanco/bibliopiscinas-y-biblioplayas-refrescantes-servicios-de-extension-cultural

${ }^{67}$ La Biblioteca Municipal de Albatera (Alicante) ha recibido distinciones por su servicio de biblioparque en los Premios a la Calidad e Innovación en la Administración Local en la Provincia de Alicante (2008) http://formacion.ladipu.com/especiales/unidadcalidad/ CALIDAD /08/AYTOS/ 06_01_MemoriaAlbatera.pdf

${ }^{68}$ Destacamos los casos de la Biblioteca Pública del Estado de Santiago de Compostela http://www.europapress.es/cultura/noticia-galicia-bibliomercado-llevara-servicios-bibliotecaanxel-casal-praza-abastos-santiago-20110901112852.html o el de las Bibliotecas Municipales de San Javier (Murcia) http://www.bibliotecaspublicas.es/sanjavier/colabora.htm\#64606, entre otros

${ }^{69}$ Destacamos el servicio de Bibliometro de Madrid, centrado en el préstamo gratuito de libros y en la puesta en marcha de campañas de animación a la lectura en el interior dlemetropolitano http://www.metromadrid.es/es/viaja_en_metro/Bibliometro_y_Locales_de_Ensayo/Bibliometro/

${ }^{70}$ Muy interesante la propuesta Bibliotren en Cataluña. Es una campaña de fomento de la cultura que consiste en convertir diez vagones de Ferrocarrils de la Generalitat de Catalunya en una biblioteca virtual. El usuario tendrá acceso a los primeros capítulos de una selección de cuarenta títulos publicados por Debolsillo y Rosa dels vents. La lectura de estas primeras páginas se podrá hacer en dispositivos móviles a través de códigos $\mathrm{QR}$ http://www.fgc.cat/esp/bibliotren.asp

${ }^{71}$ Muy sugerentes han sido los proyectos Recetas de Lectura y Biblioteca de Cabecera puestos en marcha en farmacias hospitales de la Junta de Extremadura http://www.hoy.es/prensa /20061111/sociedad/farmacias-ofreceran-recetas-lectura 20061111.html

${ }^{72}$ Las bibliotecas de instituciones penitenciarias en España. Aproximación a las bibliotecas de centros penitenciarios y sus servicios [En línea]. Madrid: Ministerio de Cultura, 2011 http://www.mcu.es/libro/docs/MC/Observatorio/pdf/Observatorio_BiblioInstPenitenciarias.pdf

73 Por ejemplo, la bebeteca de la Biblioteca Municipal de Illescas (Toledo) http://www.bibliotecadeillescas.com/07_sala_infantil_05.html y la de la Biblioteca Pública del Estado de Albaceta http://www.bibliotecaspublicas.es/albacete/seccont_36000.htm

${ }^{74}$ No podemos olvidarnos del servicio Telebiblioteca que ofrecen las Bibliotecas de la Comunidad de Madrid ofrecido a personas mayores de 70 años e impedidas que no pueden desplazarse hasta la biblioteca http://www.telebiblioteca.es/ Otras bibliotecas que ofrecen un servicio similar son la Biblioteca Municipal de Onda (Castellón) http://www.bibliotecaspublicas.es/ 
su presencia y llega a los usuarios de manera diferente y, en algunos casos, llamativa. Algunos servicios bibliotecarios podemos encontrarlos disponibles las 24 horas del día ${ }^{75}$. Sucede, como vimos anteriormente, con los servicios ofrecidos a través de Internet, pero también con determinados servicios bibliotecarios físicos en determinadas épocas del año. Es muy difícil encontrar otro servicio público, y privado, gratuito, accesible a todos, con enfoque social, y con más horas de apertura.

Otra forma de implicarse con su comunidad es ofreciendo servicios orientados al usuario, servicios adaptados que responden a demandas particulares y que permiten a la biblioteca pública amoldarse y ofrecerse eficazmente a sus usuarios, ya sea ofreciendo recursos y servicios para colectivos específicos, por ejemplo, podemos destacar el servicio bibliotecario para la comunidad sorda que ofrecen las Bibliotecas Municipales de A Coruña ${ }^{76}$, ya sea acogiendo para fines concretos a grupos de usuarios, como hace por ejemplo la Biblioteca Municipal de Eibar que ofrece sus instalaciones como centro de prácticas de idiomas para estudiantes de Filología ${ }^{77}$, ya sea implicando a los usuarios en la gestión bibliotecaria, mediante Asociaciones de Amigos como hacen en la Biblioteca Pública Provincial de Huelva ${ }^{78}$ o con los Embajadores de la Biblioteca como está previsto poner en marcha en las Bibliotecas Municipales de San Javier (Murcia) ${ }^{79}$, ya sea ofertando campañas de marketing que permitan llegar a los destinatarios de una manera diferente y sugerente, como la que ha puesto en marcha recientemente la Biblioteca Municipal de La Mina de San Adriá de Besós (Barcelona) con su campaña “....y un jamón”"80.

\section{CONCLUSIONES: REFRESQUEMOS NUESTRAS BIBLIOTECAS CON IDEAS INNOVADORAS}

"En un momento de restricciones económicas, invertir en bibliotecas es invertir en aprendizaje y cohesión social"s1

En este trabajo hemos ofrecido un muestrario de experiencias de trabajo bibliotecario que pueden considerarse innovadoras y creativas. No son todas las

\footnotetext{
onda/servicios.htm\#42172 o la Biblioteca Municipal de Valdepeñas de Jaén http:// www.bibliovaldejaen.com/2011/10/libros-casa-biblioteca-domicilio.html

75 Relación de bibliotecas públicas de Madrid abiertas 24 horas disponible en $\mathrm{http} / / /$ www.madrid.org/bpcm/servlet/Servidor?opcion=Documento\&nombre=24horas_200709_lista.pdf

${ }^{76}$ Véase http://www.coruna.es/bibliotecas

${ }^{77}$ Véase http://www.elcorreo.com/vizcaya/v/20121209/guipuzcoa/biblioteca-acogeragrupos-practica-20121209.html

${ }^{78}$ Véase http://amigosbibliotecahuelva.wordpress.com/

${ }^{79}$ Véase http://www.bibliotecaspublicas.es/sanjavier/proyectos.htm\#75206

${ }^{80}$ Véase http://sorprendemos.com/consultoresdocumentales/?p=4736

${ }^{81}$ Ferrán Civil, vicepresidente de la Diputación Provincial de Barcelona. Diario El País, edición de 21/03/2012 http://ccaa.elpais.com/ccaa/2012/03/20/catalunya/1332279094_149810.html
} 
que son, pero sí son todas las que están, es decir, las que sabíamos tras consultar literatura especializada, foros profesionales, contactos personales, etc. y de las que hemos tenido conocimiento. Seguro que habrá muchas más. Asimismo hemos dejado constancia de que la innovación es un elemento clave en la gestión de cualquier organización y que en esta innovación suele ocupar un lugar destacado la tecnología. Junto a ello, cabe resaltar a los profesionales bibliotecarios, sus métodos y enfoques de trabajo, su forma de llegar a la ciudadanía y sus propuestas de servicio. De estas experiencias de innovación puede vislumbrarse el afán de superación del colectivo bibliotecario y de no estancarse en lo ya conocido y llevado a cabo, sino en centrarse en buscar nuevas vías de servicio, nuevas propuestas de trabajo y nuevos procedimientos de fidelización de usuarios superando resistencias, convirtiendo amenazas en oportunidades y ofreciendo nuevas razones para seguir siendo útiles y necesarias en su comunidad.

El modelo bibliotecario de los últimos diez o quince años comienza a estar agotado y es necesario reinventarse, readaptarse y renovarse prestando servicios orientados al usuario, centrados en el usuario, servicios que en muchos casos resultarían impensables hace unos años y que ahora, las circunstancias y el contexto de cada comunidad, puede hacer posibles. Nuestros usuarios necesitan de bibliotecas mixtas, híbridas, en las que tengan cabida los servicios de proximidad que las ha definido desde siempre y que cuentan con una gran aceptación e interés (cuentacuentos, préstamo documental a domicilio, acceso a Internet, dinamización cultural,...) junto a nuevas dinámicas de servicio que sorprendan gratamente al ciudadano y permitan a estas instituciones seguir siendo un referente en su comunidad.

\section{REFERENCIAS BIBLIOGRÁFICAS}

BRAVO JIMÉNEZ, Pedro A.: "Set biblioteques públiques, vuit progrmes innovadors" [En línea]. Item, $\quad \mathrm{n}^{\circ} 49 \quad$ (2008), $\quad 31-44$ http://bibgirona.net/salt/massagran/planes/item_49.pdf

ECHEVERRÍA, Javier: "Avanzando en la sociedad del conocimiento: ¡innovemos!" [En línea]. Telos: Cuadernos de Comunicación e Innovación, $\mathrm{n}^{\mathrm{0}} 77$ (2008) http://sociedadinformacion.fundacion.telefonica.com/telos/ articuloautorinvitado.asp@idarticulo=2\&rev=77.htm

FENOLL CLARABUCH, Carme y LLUECA FONOLLOSA, Ciro: "Cincuenta ideas para sorprender desde la biblioteca pública" [En línea]. BiD, nº17 (2006) http://www.ub.edu/bid/17fenol2.htm

GALLO LEÓN, José Pablo: "Innovación en los servicios de bibliotecas públicas orientadas al usuario. El caso de la Biblioteca Regional de Murcia". El Profesional de la Información, vol.17, nº2 (2008), 144-154

GARCÍA GÓMEZ, F. Javier y DÍAZ GRAU, Antonio: “¿Crisis? no, gracias: recetas bibliotecarias en tiempos difíciles: la experiencia de las bibliotecas 
municipales de San Javier (Murcia)" [En línea]. Mi Biblioteca, n²8 (2012), 37$43 \mathrm{http}: / /$ eprints.rclis.org/handle/10760/16607

JANTZ, RONALD C.: "Innovaciones tecnológicas en la biblioteca: proyectos digitales que ofrecen nuevas oportunidades al bibliotecario y a la biblioteca" [En línea]. Boletín de la Asociación Andaluza de Bibliotecarios, nº67 (2002), 61-69 http://www.aab.es/pdfs/baab67/ 67a4.pdf

KNECHT CRISTÓBAL, Almudena: "La búsqueda de la innovación en proyectos bibliotecarios: el valor de la colaboración interdisciplinar" [En línea]. Scire, vol.14, n² (2008), 107-118 http://ibersid.eu/ojs/index.php/ scire/article/view/1753

LOZANO DÍAZ, Roser: "Gestión del cambio y cultura organizacional en la biblioteca pública" [En línea]. Educación y Biblioteca, nº170 (2009), 101-106 http://gredos.usal.es/jspui/bitstream/10366/108935/1/EB21_170.pdf

LOZANO DÍAZ, Roser: "Innovación en bibliotecas públicas: algo nuevo, algo útil, algo de calidad" [En línea]. El profesional de la información, vol. 17, $\mathrm{n}^{\circ} 2$ (2008), 129-134 http://www.elprofesionaldelainformacion.com/contenidos/ 2008/marzo/01.pdf

ROMERA IRUELA, María Jesús: “Orientaciones para una innovación en las bibliotecas públicas de la provincia de Segovia basada en las necesidades de información de los inmigrantes" [En línea]. El profesional de la información, vol. 17, $\mathrm{n}^{\mathrm{o}} 2$ (2008), 155-164 http://www.elprofesionaldelainformacion.com/ contenidos/2008/marzo/04.pdf

VARELA OROL, Concha: "La gestión de la tecnología en las bibliotecas" [En línea]. Boletín de la Asociación Andaluza de Bibliotecarios, n94/95 (2009), 27-45 http://www.aab.es/pdfs/baab9495/94-95col02.pdf. 